\title{
CUERPO, FEMINIDAD Y CONSUMO: EL CASO DE JÓVENES UNIVERSITARIAS ${ }^{1}$
}

\section{BODY, FEMININITY AND CONSUMPTION: THE CASE OF YOUNG COLLEGE STUDENTS}

\section{Tania Cuevas Barberousse*}

\section{RESUMEN}

Este artículo analiza las interrelaciones existentes entre la percepción corporal, la construcción de feminidad y el consumo en un grupo de mujeres jóvenes de la Universidad de Costa Rica. Se evidencian cierto número de prácticas y discursos relacionados con el cuerpo femenino en la sociedad costarricense contemporánea, y se estudian las percepciones y significados que las mujeres construyen acerca de sus cuerpos y de su feminidad.

PALABRAS CLAVES: PERCEPCIÓN * JUVENTUD * MUJERES * FEMINIDAD * CONSUMO * IDEALES ESTÉTICOS

\section{ABSTRACT}

This article analyzes a set of relationships between body perception, the construction of femininity, and consumption practices in a group of young female students from the University of Costa Rica. It assesses a number of practices and discourses associated to the female body in contemporary Costa Rican society, and considers the perceptions and meanings that women construct in relation to their body and femininity.

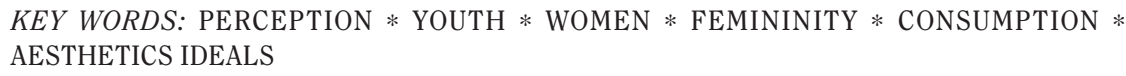

$1 \quad$ Este artículo proviene de la investigación "Consumo, percepción corporal y construcción de la feminidad: el caso de mujeres jóvenes universitarias (2007)" — distinguida con honores — para obtener el grado de Licenciatura en la Escuela de Antropología de la Universidad de Costa Rica.
* Asistente en docencia de diversos cursos en Antropología de la Universidad de Costa Rica (2000-2005). tania.cuevas@gmail.com




\section{INTRODUCCIÓN}

En la sociedad occidental actual existe una gran preocupación por el cuerpo y la salud. La mayoría de personas, especialmente las más jóvenes, sienten una gran presión social por conseguir un cuerpo bello, delgado $y$ joven, $y$ aunque este ideal es casi siempre inalcanzable, se considera incluso una condición de éxito social. Estar bello y sano ha dejado de ser una aspiración para convertirse en una especie de tiranía (Díaz et ál., 2006). Esta preocupación por la belleza física $y$ la salud tiene incluso un nombre conocido: el culto al cuerpo, que algunos autores, como Díaz (2006), han llamado somatocentrismo, del griego somas, sómatos, "cuerpo", un término que indica que el cuerpo se ha convertido en el centro de la vida. Además existe, asociado a lo anterior, lo que podemos llamar un culto a la salud. La aspiración por el bienestar psíquico y físico se encuentran por lo tanto en el centro de las aspiraciones contemporáneas.

Tomando en cuenta lo antes mencionado, se considera que el consumo es una de las vías principales por medio de canalización de esas aspiraciones en las sociedades occidentales, en la medida en que la búsqueda de la belleza y la salud se nutre del optimismo y las promesas que fomenta el mercado. El imaginario de la belleza, sobre todo la femenina, se ha convertido en un verdadero negocio, el cual define muchas de las ideas y percepciones existentes acerca del cuerpo, especialmente el de la mujer.

Este artículo analiza las interrelaciones existentes entre la percepción corporal, la construcción de feminidad y el consumo en un grupo de mujeres jóvenes de la Universidad de Costa Rica con edades comprendidas entre los 18 y los 25 años. En este sentido, se evidencian cierto número de prácticas y discursos relacionados con el cuerpo femenino, y cómo el consumo, analizado en relación con la salud y la estética, se convierte en una de las principales formas de expresión de ese culto al cuerpo y a la salud.

\section{CONSIDERACIONES TEÓRICAS}

Se parte del supuesto según el cual el cuerpo es, además de un producto biológico, una construcción social y, como tal, responde a un determinado momento histórico y a un contexto cultural particular. En ese sentido, Lagarde (1990) sostiene que la imagen corporal debe entenderse como parte de un sistema de relaciones sociales definidas culturalmente, las cuales nos ayudan a entender la manera en que las personas perciben, describen y modifican su cuerpo.

En esa misma línea de pensamiento, Le Breton (1995) sostiene que cada sociedad esboza, al interior de su visión de mundo, un saber singular sobre el cuerpo, otorgándole de esa manera sentido y valor. Para el autor, las concepciones del cuerpo son tributarias de las concepciones de la persona en la medida en que las representaciones sociales le asignan al cuerpo una posición determinada dentro del simbolismo general de la sociedad. El cuerpo es, por lo tanto, una construcción simbólica, no una realidad en sí mismo:

Sin el cuerpo que le presta un rostro, el hombre no existiría. Vivir consiste en reducir continuamente el mundo al cuerpo, a través de lo simbólico que este encarna. La existencia del hombre es corporal. Y el análisis social y cultural del que es objeto, las imágenes que hablan sobre su espesor oculto, los valores que lo distinguen, nos hablan también de la persona y de las variaciones que su definición y sus modos de existencia tienen en diferentes estructuras sociales. (1995: 7).

En el caso de las sociedades occidentales, Le Breton (1995) sostiene que la concepción del cuerpo que se admite con mayor frecuencia encuentra su formulación en el desarrollo de una estructura individualista del campo social. Sólo en ese tipo de estructuras sociales el cuerpo puede pensarse como elemento aislable del individuo, en las cuales las personas están separadas unas de otras y son relativamente autónomas en sus iniciativas y en sus valores. Funciona, de acuerdo con lo anterior, como un límite fronterizo que define, ante los otros, la presencia del sujeto.

Esta concepción está basada en una noción particular de la persona que le permite 
decir al sujeto "mi cuerpo", utilizando como modelo el de la posesión. Para Le Breton (1995), esta representación nació de la emergencia y del desarrollo de condiciones culturales específicas en las sociedades occidentales a partir del Renacimiento. Según el autor, para que la individualización a través de la materia es decir, del cuerpo, sea aceptable en el plano social, habrá que esperar el desarrollo del individualismo que opera a partir del siglo XV en Europa. En ese momento, el cuerpo se convierte en la frontera precisa que marca la diferencia entre una persona y otra, convirtiéndose en un blanco de intervención específica, como por ejemplo el de la investigación anatómica a través de la disección del cuerpo.

Esta actitud hacia el cuerpo se relaciona con el retroceso y el abandono de la visión teológica de la naturaleza que había dominado durante toda la Edad Media. Según el mismo autor, en las sociedades de tipo comunitario, en las que el sentido de la existencia implica un juramento de fidelidad al grupo, al cosmos y a la naturaleza, el cuerpo no existe como elemento de individualización ya que la persona no se distingue del grupo. Como mucho, es una singularidad dentro de la armonía diferencial del grupo.

A la inversa, el aislamiento del cuerpo en las sociedades occidentales nos habla de una trama social en la que el ser humano está separado del cosmos y de los otros. De esa manera, la distinción del cuerpo es por lo tanto la herencia histórica del hecho de que la concepción de persona haya sido aislada del componente comunitario y cósmico, y el efecto de la ruptura que se operó en el ser humano.

El cuerpo se construye por lo tanto culturalmente y es resultado de determinadas condiciones sociales y culturales en las sociedades occidentales. Es por lo tanto esencial hacer evidente los estándares de belleza prevalecientes en la actualidad para entender prácticas $y$ discursos relacionados con el cuerpo que desembocan en una determinada imagen corporal, la cual se entiende como la visión subjetiva que cada persona tiene de su cuerpo.

En el caso del cuerpo femenino, esos discursos y prácticas suponen, además, una construcción simbólica particular alrededor de lo que significa ser mujer $y$ de lo que se considera como femenino. Como ya lo afirmara Simone de Beauvoir en su obra El segundo sexo:

No se nace mujer, llega una a serlo. Ningún destino biológico, físico o económico define la figura que reviste en el seno de la sociedad la hembra humana: la civilización en conjunto es quien (la) elabora (1987: 13, citado por Pacheco, 2001: p. 39).

En ese sentido, el rol femenino asignado se debe pensar por lo tanto como dinámico, maleable y cambiante. Paralelo a la idea que se construye alrededor del rol social de las mujeres, se construye la percepción del cuerpo femenino (Pacheco, 2001). La distancia entre la diferencia sexual biológica y la construcción de las identidades, conductas, funciones y deseos de género y sexuales está marcada, por lo tanto, por la cultura.

De esa manera, la percepción que tienen las mujeres de sus cuerpos y la idea que construyen acerca de su feminidad, constituyen facetas dinámicas e inseparables de un mismo proceso, a saber, la construcción de su subjetividad. Paul Schilder (1950), uno de los pioneros en el estudio de la experiencia corporal desde la sociología y la psicología, define a la percepción corporal como la imagen mental que nos hacemos de nuestro cuerpo, entendiendo esa imagen mental no sólo como una construcción cognitiva, sino también como el reflejo de nuestras actitudes y relaciones sociales.

En ese sentido, si nuestras actuales concepciones del cuerpo están vinculadas con el ascenso del individualismo como estructura social y con la emergencia de un pensamiento racional positivo y laico sobre la naturaleza, en el caso de las mujeres, podríamos agregar, están sujetas a un sistema de dominación patriarcal, —que supone relaciones desiguales entre hombres $y$ mujeres-, $y$ a una cultura de masas y de consumo, entendidas en su versión capitalista (Le Breton, 1995). El cuerpo se convierte entonces en una especie de "objeto" que podemos modificar, y los significados que se le atribuyen son el reflejo de las actitudes hacia 
los individuos y la sociedad en general. En las sociedades occidentales, la existencia del individuo como entidad separada se acepta como realidad objetiva, aunque esta visión es de hecho bastante reciente en la historia.

La percepción corporal es, entonces, subjetiva $y$ se encuentra constantemente determinada por la experiencia social. Las mujeres experimentan y entienden sus cuerpos de acuerdo con imágenes corporales que son culturalmente construidas y definidas. En el caso que nos ocupa, el contexto al cual se alude se enmarca en los procesos actuales de globalización económica, social y cultural de los modelos de vida occidentales. Según Seremetakis (2001), la globalización ha sido profundamente estudiada desde los procesos sociopolíticos y económicos, pero existen otros nichos de la vida cotidiana poco explorados en donde la globalización ha tenido efectos profundos, como el imaginario social acerca de la belleza.

En ese sentido, Arroba (1996) argumenta que en el proceso de globalización contemporánea, muchas de las modas y tendencias vigentes en Estados Unidos se manifiestan también en sociedades consumistas como la costarricense. En ese sentido, en una escala menor, patrones similares pueden ser detectados en la construcción de género de lo femenino. Por lo tanto, el concepto de belleza debe entenderse como el producto de una evolución histórica particular, y los ideales estéticos imperantes, como la delgadez, como fenómenos históricos relativamente recientes.

Es importante mencionar que junto a la belleza, la salud es otro de los conceptos claves que representan valores supremos de nuestra época. Para Díaz (2006), en la sociedad occidental actual, la cultura del culto al cuerpo se ha apropiado de un concepto de salud entendido en forma muy amplia, alejándose de su sentido estricto (entendido como "el buen estado del organismo"), identificándose con lo bello, lo delgado, lo joven y lo equilibrado. Así, se insiste en la idea de salud interior (por dentro) y exterior (por fuera), $y$ se entiende más ampliamente como bienestar, el cual se refiere a la agilidad corporal, el equilibrio, el placer, la relajación, la tonicidad, la delgadez y la imagen bella. La salud se entrelaza así con el culto a la belleza, los cuales aparecen como dos ideales inseparables: la belleza es salud.

Esta promoción del culto a la salud y la belleza supone una dimensión de control y dominación sobre el cuerpo de las mujeres, es decir, se traduce en relaciones de poder. Arroba (1996) sostiene que vistos históricamente, el ideal de belleza femenina y la normalización del cuerpo de la mujer, tal vez sean la única opresión de género que se autoejecuta, aunque en diferentes grados $y$ en diferentes formas, a lo largo de líneas de edad, raza, clase o preferencia sexual, lo cual debe ser reconocido como una estrategia durable y flexible de control social.

Michel Foucault fue uno de los primeros teóricos modernos en situar al cuerpo en el centro de las regulaciones sociales, dentro de las cuales incluyó tanto el control ideológico como físico. Piedra (2004), discutiendo el enfoque de Foucault, sostiene que:

El cuerpo ocupa el lugar central desde donde se puede ejercer el poder. Hay muchos aspectos que se le exigen a un cuerpo, desde el ideal físico hasta la explotación de sus máximas capacidades en busca de una eficacia total. (...) Nuestro cuerpo se convierte en un instrumento importante a disciplinar. Son cuerpos dóciles que serán moldeados, sometidos, utilizados, transformados $y$ perfeccionados. (...) La disciplina es un aspecto y rasgo propio de la sociedad contemporánea (2004: 128).

Esas relaciones de poder, tomando como referencia los planteamientos anteriores, se manifiestan en dos niveles: un control social, asociado a las pautas culturales y momentos históricos que se manifiesta en múltiples componentes, incluido el corporal, $y$ un autocontrol, que correspondería a una vigilancia individual relativamente independiente del control social, tomando en cuenta que la asunción de valores es idiosincrásica y se reinterpreta de acuerdo con la situación particular e historia de vida de cada mujer.

De acuerdo con lo anterior, se considera que el consumo, entendido en su versión capitalista, es una de las vías a través de las cuales 
se expresan esas relaciones de poder y por las cuales se canaliza la idea de feminidad $y$ de ser mujer. Si bien es cierto hay múltiples formas de expresar la feminidad, se propone que a través del consumo es una de las formas más palpables y actuales. En ese sentido, Falk (1997) concuerda que el consumo moderno es el espacio principal de la construcción del ser, el cual ofrece el material para la construcción social y personal de su individualidad.

Siguiendo la propuesta teórica de Zukin (2004), el consumo puede entenderse como una actividad simbólica que se aplica no sólo a bienes, artículos y servicios, sino también a representaciones sociales acerca de la mujer. Aunque no se trate de artículos materiales, el intercambio simbólico propio de la práctica del consumo se extiende también al conjunto de ideas que convergen en las prácticas de consumo.

Para la autora, el surgimiento de una cultura de consumo, la cual se entiende como un producto distintivo de la modernidad, se relaciona con la creación moderna de lo que algunos han denominan un "ser con capacidad de elección" (a choosing self). El desarrollo de este nuevo ser se vincula con el proceso de individualización, en el cual la identidad se separa de una serie de características determinadas por el nacimiento $y$ se adscribe a un proceso individual y continuo modelado por la apariencia y el rendimiento. Las personas viven vidas mucho más anónimas y racionales y los tradicionales modelos de referencia de identidad colectiva e individual se debilitan. En ese sentido, el individuo es por lo tanto "libre" de escoger su camino hacia la auto-realización.

De esa manera, en las sociedades de consumo es posible identificar esta "tendencia generalizada" en los procesos sociales y en las mentalidades, la cual homogeniza ciertos deseos, los dirige hacia el consumo de productos y crea nuevos espacios en donde esos productos pueden ser exhibidos, comprados y disfrutados.

\section{ESTUDIOS SOBRE PERCEPCIÓN CORPORAL Y CONSTRUCCIÓN DE LA FEMINIDAD}

En años recientes ha habido un renovado interés académico y popular en relación con el cuerpo. El cuerpo ha sido reconocido como un tema válido de la teoría social desde distintas perspectivas teóricas y un elemento importante de la cultura y la política modernas (Falk, 1997; Featherstone,1998; Foucault, 1998; O'Neill, 1989; Turner, 1984).

Consideramos que uno de los enfoques más interesantes es aquel que evidencia la relación entre la cultura y la percepción corporal (Dion et ál., 1972; Grogan, 1999). Bordo (1993), trazando las implicaciones sociales ligadas a la delgadez, demuestra cómo estas cambian a lo largo de la historia y cómo son, en gran medida, construcciones culturales. El cuerpo firme $y$ tonificado, por ejemplo, es representativo en la actualidad del éxito de quien lo posee, $y$ desde el punto de vista cultural, la delgadez se asocia comúnmente con la felicidad, el éxito personal, la juventud y la aceptación social.

Siguiendo con la idea anterior, algunas investigaciones se han centrado en el análisis histórico de los ideales estéticos relacionados con el cuerpo femenino presentes en las sociedades occidentales. En ese sentido, algunos trabajos han señalado la posibilidad de estudiar el cambio cultural en relación con los ideales corporales, evidenciando el vínculo entre percepción corporal y el contexto cultural en el cual se desenvuelven las mujeres, y cómo ese entorno cultural tiene una importante incidencia sobre la idea que construyen acerca de sus cuerpos (Gimlin, 2001; Orbach, 1993; Tiggeman y Rothblum, 1988). Estudios como el de Fallon (1990), por ejemplo, demuestran cómo la idealización de la delgadez es un fenómeno reciente, siendo el resultado del marketing exitoso de la industria de la moda, la cual se ha convertido en el estándar de la cultura de la belleza en las sociedades occidentales contemporáneas.

Otro foco de interés en el análisis del cuerpo ha sido el estudio de la construcción social de la feminidad, $y$ cómo esta se encuentra vinculada con la percepción del cuerpo femenino (Edut, 1998; Orbach, 1993; Passerinni, 1993; Wolf, 1991). En ese sentido, Chapkis (1986) argumenta que la mujer se encuentra oprimida por una máquina cultural global (construida a partir de la publicidad, los medios de comunicación y la industria cosmética), la cual promueve 
un estrecho ideal de belleza occidentalizado a escala mundial.

Estudios antropológicos sobre el cuerpo han provisto una perspectiva cultural comparativa que toma en cuenta los valores y creencias de individuos de sociedades y culturas distintas (Mead, 1975; Pesenti, 1984; Sault, 1994). El interés por estudiar la construcción social del cuerpo y de la feminidad desde la antropología radica en el hecho de hacer evidente el carácter histórico y cultural de tales construcciones, analizando no sólo el contexto, sino también la manera en que cada mujer resignifica ese contexto. Es importante mencionar, dentro de este campo, a Le Breton (1995) y su obra Antropología del cuerpo y modernidad, en la cual se realiza un estudio del mundo moderno desde una perspectiva antropológica y sociológica cuyo hilo conductor es el cuerpo.

Según González Suárez y Guzmán Stein (1994), los primeros trabajos sobre la mujer realizados en Costa Rica fueron elaborados por hombres, cuyos contenidos eran una clara expresión de enfoques androcéntricos sobre la mujer. Durante la primera mitad del siglo xx, los asuntos de la mujer fueron ignorados por la mayoría de las disciplinas. Al inicio de los años setenta se empieza a reconocer a la mujer como objeto de estudio. Se construyeron hipótesis y propuestas alrededor de la dependencia y la vulnerabilidad femeninas, contribuyendo con ellos a su mayor marginación.

A partir de entonces se han realizado varias investigaciones en ese sentido (Arroba, 1996; Salazar, 2007). Un ejemplo es el trabajo de Mora, Muñoz y Villareal (2001) sobre la imagen corporal en adolescentes estudiantes del Liceo Monseñor Rubén Odio Herrera ubicado en San José. En su investigación, las autoras recogen experiencias de las jóvenes respecto a la forma de vivir su feminidad en la adolescencia, profundizando en las percepciones y significados que, a través de la educación, ellas han construido alrededor del cuerpo femenino.

Tomando en cuenta la producción literaria anteriormente mencionada, podemos afirmar que existe una preocupación general en torno al modo en que la promoción constante de la delgadez y de cierto tipo de modelo feme- nino afecta la percepción que tienen de sus cuerpos las mujeres a las cuales va fundamentalmente dirigida la publicidad: las jóvenes, potenciales consumidoras de los bienes producidos por las industrias de la cosmética, la moda, la medicina y la alimentación, las cuales mueven al año miles de millones de dólares. De ahí el interés principal por realizar una investigación de este tipo en el contexto de la sociedad costarricense que aborde, desde una perspectiva antropológica, las percepciones que construye un grupo de mujeres sobre ellas mismas, en términos de sus corporeidades, y las relaciones de estas con la construcción de su feminidad y la influencia del contexto en el cual se desenvuelven.

\section{METODOLOGÍA}

Este trabajo combina métodos de investigación cuantitativos y cualitativos con el propósito de descifrar y evidenciar las vinculaciones que existen entre los significados e interpretaciones construidos por un grupo de mujeres en torno a su cuerpo y su feminidad, y cómo estas reconstrucciones se vinculan con prácticas específicas de consumo en un contexto determinado.

Se aplicaron dos técnicas de recolección de datos: un cuestionario y entrevistas a profundidad. El número de casos utilizados fue determinado con base en tres factores, siguiendo el planteamiento de Hernández et ál. (2006): 1) la capacidad operativa de recolección y análisis; 2) el número de casos que, a nuestro juicio, nos permitieron responder a las preguntas de investigación planteadas (principio de la "saturación de categorías") y 3) la frecuencia y accesibilidad de los casos bajo estudio.

Como punto de partida, se aplicó un cuestionario entre los meses de febrero y marzo del 2006, a 100 mujeres universitarias con edades comprendidas entre los 18 y los 25 años, del área de Ciencias Sociales, el área de Salud y el área de Ingeniería. El objetivo de este cuestionario no fue el de establecer resultados que pretendieran ser representativos, sino el de recoger información general sobre las prácticas de consumo de esas mujeres en relación con la 
salud y la estética y la incidencia de esas prácticas en la construcción de sus feminidades y las percepciones de sus propios cuerpos. A partir de los resultados arrojados por el cuestionario, los cuales se procesaron con el programa SPSS, se construyeron categorías de análisis con respecto a las ideas acerca del cuerpo femenino $y$ construcción de la feminidad de ese grupo de mujeres.

En un segundo paso, durante el mes de octubre del mismo año, se escogieron seis mujeres que previamente habían respondido al cuestionario para trabajar con ellas entrevistas a profundidad. El objetivo de las entrevistas fue el de analizar con mayor detalle, desde sus propias experiencias, las relaciones existentes entre la percepción de sus cuerpos, la construcción de sus feminidades y sus prácticas de consumo. Estas mujeres fueron seleccionadas aleatoriamente tomando en consideración su carrera con el propósito de contar con representantes de cada área de estudio.

\section{RESULTADOS}

\section{DATOS PERSONALES}

Un total de 100 estudiantes respondieron al cuestionario, con edades comprendidas entre los 18 y 25 años. Todas las edades estuvieron representadas de manera bastante equitativa, con un ligero predominio de estudiantes con edades entre los 20 y 22 años. Del total, 40 estudiantes correspondieron al área de Ciencias Sociales (incluyendo las carreras de Antropología, Comunicación Colectiva, Historia, Psicología, Sociología y Trabajo Social), 31 al área de Salud (entre Farmacia, Medicina, Microbiología, Nutrición, Odontología, Salud Ambiental y Terapia Física), y 29 al área de Ingeniería (Civil, Industrial, Mecánica, Química y Topográfica).

Del total de mujeres que respondieron al cuestionario, sólo 18 de ellas trabajaban, lo que significa que la gran mayoría, a saber, las 82 restantes, no tenían trabajo al momento de responder al cuestionario. De esas 82 estudiantes, 79 vivían con sus padres, quienes proveían el dinero para sus gastos personales. De las mujeres que trabajaban, 9 ganaban menos de 100 mil colones al mes, 5 entre 100 y 200 mil, 3 entre 200 y 300 mil y sólo una ganaba entre 300 y 400 mil. Nótese que la mayoría de mujeres que trabajaban contaban con un promedio mensual no mayor a los 200 mil colones. Aún así, como veremos más adelante, los gastos relacionados con la estética fueron importantes para la gran mayoría de mujeres, independientemente de su situación laboral.

En cuanto a las seis mujeres a las que se le realizaron entrevistas a profundidad, la mitad de ellas no trabajaban. Entre las que lo hacían, sus salarios mensuales eran de 135, 150 y 200 mil colones respectivamente. Por área de estudios se entrevistaron a dos estudiantes de antropología, una de sociología, una de comunicación colectiva, una de medicina y una de ingeniería industrial. Sus edades oscilaban entre los 20 y 24 años.

\section{AUTOPERCEPCIÓN CORPORAL}

En esta sección se discute el tema de la autopercepción corporal de las estudiantes que participaron en el estudio. Para analizarla se tomaron en cuenta las categorías siguientes: hábitos alimenticios, hábitos deportivos y percepción corporal.

\subsection{HÁBITOS ALIMENTICIOS}

La relación con la comida se evidenció, durante las entrevistas, como compleja y reveladora de ideas acerca de su cuerpo. Primero: para las entrevistadas, el autocontrol es fundamental a la hora de comer y no sólo se debe controlar el tipo de alimento que se ingiere, sino también sus cantidades. Por ejemplo, una de las entrevistadas comentó: "Lo importante es comer bien y equilibrado. Si uno comió mucho en el almuerzo, no comer tanto en la cena". Segundo: se aprecia una especie de "satanización" de las grasas y la gordura, lo que se evidencia en los comentarios de una de las entrevistadas: "me da asco cuando se cocina con mucho aceite, mantequilla o por ejemplo los chicharrones, empanadas, todo ese tipo de 
cosas". Tercero: junto a la idea de autocontrol, la idea de castigo se expresa cuando se considera que se han excedido las cantidades apropiadas de alimento, $y$ en este caso se traduce en ejercicio físico, evidenciando la relación entre el ejercicio y la comida como formas de controlar y modificar el cuerpo. Una de las entrevistadas comentó al respecto: "Si me como un helado, lo que hago es que me subo a la bici una hora más...salada...lo que hay que hacer es cuidar lo que uno come, no excederse, no mezclar carbohidratos y no comer nunca comida rápida". Cuarto: ideas como "no hay que mezclar los carbohidratos" y "comer equilibrado" nos remiten a expresiones de tipo médicas que se utilizan para legitimar el control que se debe ejercer a la hora de comer.

Es importante señalar que estas actitudes las expresaron todas las mujeres entrevistadas, sin distinción de edad, carrera o poder adquisitivo. Como veremos más adelante, estas no son aisladas, sino que forman parte de todo un conjunto de ideas y prácticas bastante generalizadas entre las mujeres que participaron en el estudio.

Un ejemplo de lo anterior lo constituye el hecho de que 67 de las mujeres que respondieron al cuestionario habían modificado alguna vez sus hábitos alimenticios. La razón principal de esta modificación tuvo que ver, para 41 de ellas, con razones estéticas. Además, 39 de ellas lo habían hecho regularmente. Es importante puntualizar que, en el caso de cada una de las tres áreas de estudio, las mujeres que habían modificado sus hábitos alimenticios fueron siempre mayoría con respecto al total de estudiantes encuestadas dentro de esa área. Si tomamos en cuenta la edad, la misma tendencia persistió.

\subsection{HÁBITOS DEPORTIVOS}

Junto con las actitudes hacia la comida y los hábitos alimenticios asociados a ellas, el ejercicio físico fue otro de los medios utilizados para ejercer control sobre el cuerpo $y$ modificarlo. Lo interesante de resaltar en este apartado es la expresión de la autopercepción corporal en el tipo de ejercicios que realizaban las entrevistadas. Las actividades físicas que practicaban, el lugar donde lo hacían y los objetivos de las mismas, revelaron muchas de las ideas $y$ percepciones que las mujeres entrevistadas tenían acerca de sus cuerpos. En efecto, el ejercicio físico fue visto, en la mayoría de los casos, como un medio para lograr un cambio en la forma de ciertas áreas del cuerpo consideradas como "problemáticas" y como una actividad casi "obligatoria" para mantenerse delgada y tonificada. De las seis entrevistadas, todas realizaban algún tipo de ejercicio físico. El hecho de que practicaran alguna actividad de este tipo no es tan importante como los objetivos y el lugar donde lo hacían. Cinco de las entrevistadas asistían a un gimnasio, $y$ el objetivo principal era "estar en forma": "Para mí lo más importante del ejercicio es marcar músculos más que todo, fortalecer piernas y panza... eso es en lo que más pienso cuando voy al gym...".

A partir de los comentarios anteriores notamos que los objetivos del ejercicio físico realizado por estas mujeres se centraban en la modificación de alguna parte de su cuerpo. Nuevamente, la idea de control era el fundamento de la actividad física: se realiza porque, a través de ella, se tiene poder de modificación sobre el cuerpo. Durante las entrevistas, por ejemplo, fue interesante constatar la relación que se construía entre lo que se consideraba como saludable y lo que se percibía como estético. A la hora de hablar de los ejercicios físicos, las entrevistadas comentaban constantemente los beneficios que para la salud significaba el practicar algún tipo de ejercicio físico, aunque resultaba evidente que lo que parecía primar eran los beneficios estéticos y poder de modificación corporal de los mismos.

Los resultados obtenidos en las entrevistas se reflejaron en los resultados de los cuestionarios. De la totalidad de mujeres que lo respondieron, 54 de ellas realizaban algún tipo de actividad física, $y$ de esa actividad física, 48 practicaban ejercicios aeróbicos en un gimnasio; el objetivo de esos ejercicios era, sobre todo, modificar alguna parte del cuerpo. El hecho de que los ejercicios físicos se realizaran en su mayoría en un gimnasio es importante porque nos revela cómo este tipo de infraestructura se 
ha convertido en un lugar donde se promueven ciertos tipos de actividades físicas relacionadas directamente con anhelos estéticos que se fundamentan en interpretaciones culturales sobre el cuerpo.

\subsection{PERCEPCIÓN CORPORAL}

La relación entre el peso y la percepción corporal fue probablemente una de las más evidentes. Como ya mencionamos anteriormente, la gran mayoría de las mujeres encuestadas habían modificado alguna vez sus hábitos alimenticios por razones estéticas. En el caso de las estudiantes entrevistadas, todas habían modificado esos hábitos. Lo interesante es resaltar que las razones estéticas que fundamentaban dichas modificaciones tenían que ver con la modificación de su peso corporal. Según algunas de las entrevistadas, su peso actual debería ser menor. Así, de las seis entrevistadas, cinco consideraban que debían bajar de peso.

En el caso del cuestionario, de las 67 mujeres que habían modificado sus hábitos alimenticios, 54 de ellas lo habían hecho para bajar de peso. De esas 67 mujeres, 65 de ellas consideraban que su peso era normal. Lo anterior evidencia que mujeres que consideran su peso como normal, han cambiado sus hábitos alimenticios con el fin de modificarlo, $y$ que la tendencia de esa modificación es hacia la delgadez. De todo lo anterior, lo que se evidencia sin lugar a dudas es una insatisfacción corporal general que se expresa de distintas formas. Así, sus preocupaciones estéticas no sólo se limitaron al peso, sino también a partes específicas de sus cuerpos: "No estoy satisfecha con mi cuerpo...para nada (...) Cambiaría mi pancita que me gusta pero me quitaría grasita y quiero tonificar más las piernas... me gustaría definir más en general músculos como los brazos y la espalda...".

El querer modificar sus cuerpos fue una idea que se repitió tanto durante las entrevistas como en las respuestas de los cuestionarios. Así, de las 100 mujeres que respondieron al cuestionario, 78 de ellas consideraron que alguna parte de su cuerpo debía ser modificada.

Los pechos, el abdomen, los glúteos, las piernas y los brazos fueron las partes que, en orden de importancia, deseaban modificar. En esas partes, las modificaciones que más se mencionaron, excepto para los pechos, fueron la tonificación y la disminución del volumen o tamaño de la parte del cuerpo mencionada. Sólo en el caso de los pechos, el aumento de su tamaño pareció ser más importante que su disminución, aunque la tonificación siguió siendo primordial también para esa parte del cuerpo.

\section{IDEALES ESTÉTICOS}

La información anterior encuentra su fundamento en una serie de estereotipos e ideales con respecto al cuerpo femenino y lo que implica ser mujer (feminidad) en nuestros días. Estos debemos entenderlos insertos en un conjunto mucho más amplio de discursos sobre la feminidad, asociados a una sociedad patriarcal, que supone desigualdades entre el hombre $y$ la mujer, $y$ al ascenso del individualismo $y$ del consumismo. Ideas acerca de lo que se define como un cuerpo estéticamente bello y un cuerpo que "se ve" sano, ideas asociadas a la delgadez y la obesidad, así como de lo que se considera como femenino, nos demuestran cómo muchas de las insatisfacciones estéticas expresadas por las mujeres estudiadas tienen su punto de encuentro en una serie de representaciones mentales $y$ sociales acerca del cuerpo femenino.

\subsection{CUERPO BELLO Y CUERPO SANO: "LA BELLEZA ES SALUD"}

En el caso de las mujeres entrevistadas, podemos rescatar una idea que parece ser general ante la pregunta de cómo definían un cuerpo femenino bonito: es aquel que es proporcionado, delgado y con curvas. Además, términos mencionados como "tonificado", "trabajado" y expresiones tipo "que se vea que va al gimnasio", nos remiten directamente a los resultados del apartado anterior. Además, el "término medio" también pareció ser importante: no hay que ser muy musculoso pero tampoco muy delgado. La proporción pareció ser uno de los aspectos esenciales a la hora de definir lo que se considera como un cuerpo bonito: la belleza se 
nos presenta como la expresión de lo proporcionado y lo simétrico.

Lo interesante es que las definiciones de un cuerpo sano que expresaron las entrevistadas fueron similares a las que nos dieron para el caso de un cuerpo bonito. En ese sentido, la belleza es la expresión de la salud, del buen funcionamiento del cuerpo. Nótese la respuesta de una de las entrevistadas: "Un cuerpo sano es un cuerpo de deportista... que se vea que hace ejercicio".

A partir de lo anterior, podemos deducir que la salud se consideró sinónimo de belleza. La salud se relaciona directamente con los rasgos contemporáneos de belleza. Por el contrario, esto fue lo que nos respondieron cuando se les preguntó acerca de la obesidad: "Alguien obeso es alguien que no se cuida, que sólo come comidas grasosas, que no tiene voluntad para hacer ejercicio, ni hacer dietas".

Si la salud se asocia con la belleza, esta última se transforma por lo tanto en un imperativo moral: hay que cuidarse, se debe tratar de ser bello, legitimándose como una forma de aspiración contemporánea. La obesidad como condición física se entiende como su contrario, extendiéndose al ámbito tanto mental como moral: las personas obesas no tienen fuerza de voluntad y son personas perezosas que no se preocupan por su salud. En una cultura obsesionada con la imagen, esta parece ser el reflejo de lo que es la persona: verse bien implica "ser bueno". La imagen se convierte en la realidad misma, y la apariencia física se convierte en la medida de lo bueno y lo valioso.

\section{2. LA FEMINIDAD}

Una de las personas entrevistadas definió la feminidad de la siguiente manera: "Una mujer femenina es una mujer bien cuidadita, siempre bien vestida $y$ pintada, bonita... una mujer tranquila... que no grite mucho". Otra de las entrevistadas comentó:

- Mi mamá siempre me ha dicho que hay que estar presentable, que una mujer debe ser coqueta.
-Para mí lo femenino es una actitud: el caminar, el vestir, el comportarse, tener glamour, clase, ser delicada, cuidadoso con uno mismo... hay que saber lo que uno tiene $y$ saber mostrarlo, ser sexy pero moderado.

De acuerdo con esta respuesta, la feminidad se relacionó con la personalidad, con una actitud, una forma de ser $y$ de comportarse ente los demás. Sin embargo, en el fondo, lo que sobresale es la asociación entre la feminidad y la apariencia física. Ser femenina, además de asociarse a un comportamiento, implica la actitud siempre vigilante de la propia apariencia física. Arreglarse, saberse vestir, peinarse y maquillarse es lo que define a una "mujer femenina". Esta feminidad implica también un "cierto misterio" a la hora de "mostrarse", escogiendo adecuadamente las cualidades a resaltar. Con respecto a esto último, una de las entrevistadas afirmó: "La feminidad tiene que ver con la personalidad. Es una actitud, cómo uno camina, cómo uno se arregla... es tener cierto misterio... mostrar un poco pero no mucho...".

Una mujer femenina también se entiende como una mujer cautelosa, que sabe comportarse adecuadamente en todo tipo de situaciones y que no sea "ruidosa". Feminidad y belleza van en ese sentido de la mano. La apariencia física es, por lo tanto, el eje transversal de la mujer: esta debe ser bonita para verse sana $y$ femenina.

\section{EL CONSUMO}

En este apartado se recoge información acerca del consumo de productos y de tratamientos relacionados directamente con la estética corporal. Lo que nos interesa resaltar es cómo las ideas acerca del cuerpo y la belleza se expresan en prácticas de consumo específicas, $y$ qué tan importantes son esas prácticas para las mujeres entrevistadas, tomando en cuenta su situación económica.

El uso de maquillaje fue una de las variables que utilizamos para acercarnos a las prácticas de consumo del grupo estudiado relacionadas con la estética. Consideramos que el 
maquillaje se encuentra entre una de las mayores preocupaciones de las mujeres contemporáneas: gastan dinero, tiempo, esfuerzo y energía utilizándolo. De todas las prácticas de belleza en las sociedades occidentales, la utilización de maquillaje es tal vez uno de los componentes esenciales de la identidad femenina (Wolf, 1991). De las seis mujeres entrevistadas, todas utilizaban maquillaje, aunque algunas sólo en ocasiones especiales. Tres de ellas lo usaban diariamente. Una nos comentó: "Siempre uso... me delineo los ojos siempre... eso nunca falla... es más, nunca salgo sin los ojos delineados porque me siento fea".

Lo que se desprende de los comentarios generales de las entrevistadas es la importancia de la apariencia y del uso del maquillaje en la vida diaria de algunas mujeres. Algunas de ellas, por ejemplo, afirmaron no "sentirse ellas mismas" sin maquillaje:

-Yo no soporto andar sin maquillaje... no salgo del baño sin pintarme las cejas, porque siento que no tengo... cuando ando sin maquillaje no siento que sea yo...

Para algunas mujeres, el maquillaje se convierte en parte esencial de la construcción de la identidad femenina, $y$ es tan importante que no saldrían de la casa sin él. Así, el uso del corrector de ojeras, el delineador, el maquillaje para cejas, entre otros, las hace sentirse "cómodas" en público. Para otras, el maquillaje sólo es utilizado en ocasiones especiales, siendo una práctica esporádica. Sin embargo, independientemente de su frecuencia de utilización, lo que nos interesa resaltar es que por lo menos la mitad de las mujeres encuestadas lo utilizaban diariamente, $y$ que para ellas significaba un gasto económico relativamente importante. De acuerdo con los resultados de los cuestionarios, de la totalidad de mujeres, 76 utilizaban maquillaje, de las cuales más de la mitad lo usaban siempre.

Además del maquillaje, otro rubro importante de consumo relacionado con la estética tuvo que ver con la variedad de productos de belleza utilizados por las entrevistadas. Una de ellas nos comentó: "Yo uso productos para el pelo especiales que compro en el salón... y me hago las uñas todas las semanas...."

El gasto en productos de belleza del grupo de mujeres analizado en nuestro estudio osciló entre menos de 5 mil para 13 de ellas, entre 5 y 10 mil para 37, entre 10 y 20 mil para 27, entre 20 y 30 mil para 12 y entre 30 y 50 mil para 10. Para la mayoría, ese gasto era importante en relación con otros y todas las mujeres que participaron en la investigación tenían algún tipo de gasto que tuviera que ver con este rubro. Además, del total de mujeres, 83 de ellas consideraron que la marca era más importante que el precio. Al preguntárseles acerca de cómo se enteraron de las marcas de maquillaje que utilizaban, 74 de esas 83 respondieron que a través de los diferentes medios de comunicación, especialmente los anuncios publicitarios televisivos y en las revistas.

Además de los productos de belleza anteriormente mencionados, los tratamientos estéticos como limpiezas de cutis, masajes reductores y blanqueamientos dentales figuraron entre los gastos relacionados con la belleza del grupo de mujeres analizado. Aunque sólo 18 de ellas se los habían realizado alguna vez, es interesante resaltar que 62 mujeres de las 82 restantes se los harían si tuvieran los medios para hacerlo. Entre los tratamientos mencionados, el blanqueamiento dental y las limpiezas de cutis fueron los más importantes. Entre las mujeres entrevistadas, el blanqueamiento dental fue el más mencionado.

El deseo de realizarse este tipo de tratamientos nos parece importante en la medida en que refuerza muchas de las tendencias que hemos venido analizando. La idea del cuerpo como objeto modificable se refleja en el consumo (o deseo de consumo) de toda una variedad infinita de productos $y$ tratamientos que prometen la modificación del cuerpo y/o de algunas de sus partes.

\section{DISCUSIÓN}

A partir de los datos expuestos, reconocemos que existe un discurso dominante en torno a la belleza física, y que hoy en día existe una gran presión social por ser bella y delgada, 
independientemente de la edad que se tenga $y$ la carrera que se curse. Mediante un lenguaje dualista que opone la belleza a la fealdad, se fundamenta toda una serie de cánones estéticos que, por su naturaleza, resultan difíciles de alcanzar para la gran mayoría de las mujeres. Como lo plantea Arroba (1996), lo anterior implica el surgimiento de una (in)satisfacción corporal generalizada entre las mujeres entrevistadas y encuestadas, la cual se debe entender como subjetiva y socialmente determinada. Es importante resaltar lo anterior en la medida en que la percepción corporal del grupo analizado no dependió del peso ni de la forma de sus cuerpos, sino de la evaluación subjetiva de lo que significaba para ellas tener cierto tipo de cuerpo en el marco de una cultura particular. En ese sentido, los resultados de esta investigación muestran que la (in)satisfacción corporal del grupo analizado no está relacionada de manera objetiva ni con el peso ni con la forma del cuerpo de las participantes.

De acuerdo con los datos obtenidos, proponemos que existe una exaltación del cuerpo que se caracteriza por un culto a la imagen estética, tal y como lo afirma Díaz (2006), la cual se compone de los siguientes aspectos:

1. Una evaluación del atractivo físico en relación con ideales estandarizados de belleza;

2. Una tendencia a la uniformización de los cuerpos;

3. Una idealización de la delgadez;

4. Una disciplina marcada por normas de cuidado, control y vigilancia del cuerpo;

5. Un consumo de ciertos productos $y$ actividades relacionados con la belleza y la salud; $y$

6. Una insatisfacción corporal generalizada.

Lo anterior se traduce en la creación de ciertas conductas, costumbres y hábitos específicos, reveladores de ciertos valores culturales relacionados con la belleza y la salud. La gran mayoría de mujeres entrevistadas y encuestadas compartían ese tipo de ideas, lo cual nos permitió distinguir tendencias generales a pesar de su edad, su carrera o su poder adquisitivo.
Aunque unas gastaran más que otras en maquillaje o cremas, para la gran mayoría el gasto en este tipo de productos de belleza significó una porción importante con respecto a su presupuesto total, lo cual nos indica la manera en que las ideas sobre el cuerpo expresadas por el grupo analizado se materializan en prácticas de consumo determinadas, las cuales reflejan a su vez una forma específica de entender el cuerpo y su relación con el mundo que las rodea.

En este inicio del siglo XXI, presenciamos una innegable creciente preocupación por el cuerpo. Dietas, medicamentos y avances en campos como la cirugía plástica, han abierto las posibilidades, en el mundo occidental, de cambiar la forma en que nos vemos. Como se discutió teóricamente en la primera parte de este trabajo, la representación de un estrecho rango de ideales estéticos no deja margen de duda acerca de cómo deben lucir las mujeres. Como lo sugiere Chapkis (1986), la homogeneidad cultural de estos ideales, junto a la idea de que el poder de cambio se encuentra en nosotras mismas, ha dado paso a una palpable insatisfacción corporal $y$ al surgimiento de sentimientos de culpa. Es necesario por lo tanto un cambio sustancial en la manera en que se percibe el cuerpo, sobre todo el femenino, en las sociedades occidentales, para dar cabida a la gran diversidad estética existente y al proceso de autoafirmación de las mujeres en la sociedad contemporánea.

\section{BIBLIOGRAFÍA}

Arroba, Anna. "La construcción de la femeneidad en la era de la globalización: el caso de Costa Rica”. Ponencia presentada en el Tercer Congreso Centroamericano de Historia. San José, Costa Rica, 15 al 18 de julio de 1996.

Bordo, Susan. Unbereable weight: feminism, Western culture and the body. Berkeley: University of California Press, 1993.

Chapkis, Wendy. Beauty secrets: women and the politics of appearance. South End Press, 1986. 
Díaz, José. A; Morant, Ricard y Westall, Debra. El culto a la belleza y la salud. La retórica del bienestar. Madrid: Editoral Biblioteca Nueva, 2006.

Dion, Karen; Berscheid, Ellen y Walster Elaine. "What is beautiful is good". Journal of Personality and Social Psicology 24. 1972: 285-90.

Edut, Ophira (compiladora) y Walter, Rebecca (prefacio). Adiós, Barbie. Seattle: Seal Press, 1998.

Falk, Pasi. The Consuming Body. London: Sage, 1997.

Fallon, April. "Culture in mirror: sociocultural determinants of body image". En: T. Cash y T. Pruzinsky (editores). Body images: development, deviance and change. New York: Guilford Press, 1990.

Featherstone, Michael. Consumer Culture and Postmodernism. London: Sage, 1998.

Foucault, Michel. Historia de la sexualidad. México D.F: Siglo Veintiuno editores. 3 volúmenes, 1998.

Gimlin, Debra. Body Work: Beauty and SelfImage in American Culture. Berkeley: University of California Press, 2001.

González, Mirta y Guzmán, Laura. "Los estudios de la mujer en Costa Rica: desafiando el pasado, construyendo el futuro". Revista de Ciencias Sociales 65. Universidad de Costa Rica, setiembre 1994: 7-16.

Grogan, Susan. Body Image. Understanding body dissatisfaction in men, women and children. Londres: Routledge, 1999.

Hernández, Roberto; Fernández-Collado, Carmen y Batista, Pilar. Metodología de la Investigación. México D.F: Mc Graw Hill, 2006.

Lagarde, Marcela. Los cautiverios de las mujeres. México D.F: Coordinación General de Estudios de Posgrado, Ciudad Universitaria, 1990.

Le Breton, David. Antropología del cuerpo y modernidad. Buenos Aires: Ediciones Nueva Visión, 1995.

Mead, Margaret. Sex and Temperament in Three Primitives Societies. New York: W. Morrow, 1975.

Mora, Laura; Muñoz, Graciela y Villareal, Cecilia. "La imagen corporal en adolescentes: expresión de los aprendizajes socioculturales adquiridos sobre el ser mujer". Revista de Educación 25 (1). Universidad de Costa Rica. 2001: 111-122.

O’Neill, John. The Communicative Body. Evanston: Northwestern University Press, 1989.

Orbach, Susie. Hunger Strike: the anorectic's struggle as a metaphor for our age. London: Penguin, 1993.

Pacheco, Xenia. "Cuerpos y espacios: percepciones de las mujeres". [Tesis de Magíster Scientiae en Estudios de la Mujer]. Universidad Nacional. 2001.

Passerini, Laura. "Sociedad de consumo y cultura de masas". Historia de las Mujeres: el siglo $X X$, guerras, entreguerra y posquerra. Tomo 9. Bajo la dirección de Georges Duby y Michelle Perrot. Madrid: Taurus, 1993.

Pesenti, Marisa. "La Teoría antropológica y la perspectiva de género". La Antropología Social y los estudios de la mujer. Compiladora Estela Grassi. Buenos Aires: Editorial Humanitas, 1984. 
Piedra, Nancy. "Leyendo a Foucault desde la perspectiva de género". Revista de Ciencias Sociales 106. Universidad de Costa Rica, marzo 2004: 123-141.

Salazar, Zaida. "Imagen corporal femenina $y$ publicidad en revistas". Revista de Ciencias Sociales 116. Universidad de Costa Rica, febrero 2007: 71-85.

Sault, Nicole (editora). Many Mirrors. Body image and social relations. New Brunswick, New Jersey: Rutgers University Press, 1994.

Seremetakis, Nadia. "Toxic Beauties. Medicine, Information and Body Consumption in Transnational Europe". Social Text 6819 (3). 2001: 115-129.
Shilder, Paul. The image and the appearance of the human body. New York: International Universities Press, 1950.

Tiggemann, Marika y Rothblum, Esther. "Gender differences and social consequences of perceived overweight in the United Sates and Australia". Sex Roles 18. 1988: 75-86.

Turner, Bryan. The Body and Society. Oxford: Basil Blackwell, 1984.

Wolf, Naomi. The Beauty Myth. How image of beauty are used against women. New York: William Morrow, 1991.

Zukin, Sharon. Point of Purchase: How Shopping Changed American Culture. New York: Routledge, 2004. 